A. Pramesh Rao, G. Swarup and Gopal-Krishna, eds.

\title{
Environmental Effects and the Dynamical State of Coma from a VLA HI Survey
}

\author{
H. Bravo-Alfaro ${ }^{1}$, J. H. van Gorkom ${ }^{2}$, V. Cayatte ${ }^{3}$ and C. Balkowski ${ }^{3}$ \\ ${ }^{1}$ Dept. de Astronomía, Universidad de Guanajuato. Apdo. \\ Postal 144 Guanajuato 36000, México \\ 2 Department of Astronomy, Columbia University, \\ New York, N.Y. 10027 USA \\ ${ }^{3}$ Observatoire de Paris DAEC, 92195 Meudon Cedex, France
}

\begin{abstract}
We present results obtained from our 21cm VLA survey of spirals in Coma. The goal of this HI imaging of galaxies is twofold: first, study the interaction between individual galaxies and the cluster environment, looking for a connection with the starburst and post starburst phenomena. Second, to investigate the dynamical state of the cluster, analyzing the gas content of different groups of galaxies.
\end{abstract}

\section{Observations}

Forty two hours of the VLA, in its D configuration, were devoted in March 1999 to observe two fields in the center and SW of Coma. Additionally we dispose of previous data over 1 Abell radius of Coma $\left(\sim 1.2^{\circ}\right)$, obtained in 1995 and 1996 with the VLA in both, C and D configurations. Nineteen spiral galaxies were detected across 12 pointings (Bravo-Alfaro et al. 2000). Our typical spatial resolution is around $35^{\prime \prime}$, with a velocity resolution of $21 \mathrm{~km} \mathrm{~s}^{-1}$. The HI mass detection threshold is between $3 \times 10^{7}$ and $10^{8} \mathrm{M}_{\odot}$.

\section{The Global Properties of Coma}

We observe in the central region of Coma asymmetries in HI and even displacements of the shrunken $\mathrm{HI}$ disks near the cluster center. All the perturbed HI disks are in projection within the boundaries of the $\mathrm{X}$-ray emission suggesting that an interaction with the ICM is at work. Twelve spiral galaxies were not detected (Fig. 1), most of them around the Coma core where the distribution of detected galaxies is very non uniform: most of the detections lie east of the center, they are HI deficient and they must be falling and being stripped during this process. In the west side of the center there are almost no HI detections.

We find gas rich and gas poor groups of galaxies in Coma that can be isolated in space and velocity. The cluster HI distribution shown in Fig. 1 strengthens that gas rich galaxies are associated with the groups CGCG 160-098 and IC 4088 in the north, while a lack of $\mathrm{HI}$ is associated with the groups NGC 4944 in the east, NGC 4911 in the center, and NGC 4839 in the south. We conclude that the most Hi poor groups have gone through the center (Bravo-Alfaro et al. 2000). 


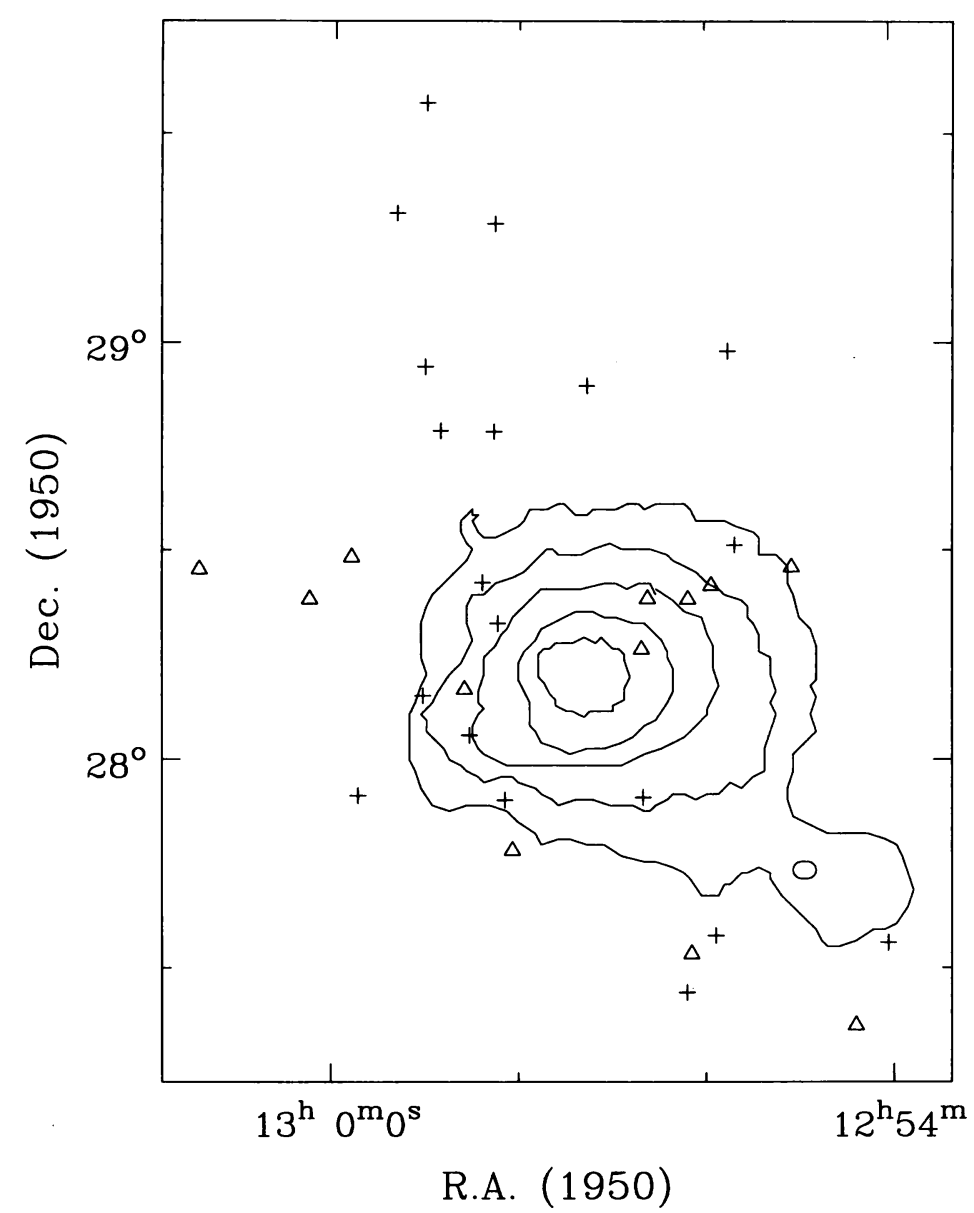

Figure 1. Position of the detected (crosses) and non-detected (triangles) galaxies in the present survey. Contours correspond to the ROSAT X-ray emission by Vikhlinin et al. (1997).

\section{The SB and PSB Galaxies}

We made a special effort to detect in $\mathrm{HI}$ the blue disk galaxies (Bothun and Dressler 1986), and the post starbursts (PSB) reported by Caldwell et al. (1993, 1997), most of them located in the SW of Coma. We detected six blue disks in $\mathrm{HI}$, but none of the PSB was detected. Our HI mass upper limit for the PSBs ranges between 3 and $7 \times 10^{7} \mathrm{M}_{\odot}$.

Most of the Hi detected starbursts (SB) are in an annular region between $0.4 \mathrm{Mpc}$ and $0.6 \mathrm{Mpc}$ from the cluster center, which coincides with the outskirts of the $\mathrm{X}$-ray emission. This is similar to what has been seen in Butcher-Oemler clusters (Dressler \& Gunn 1990), confirmed in Coma by the UV survey carried 
out by Donas et al. (1995), who reported that $38 \%$ of the UV flux is produced in a ring lying between $20^{\prime}$ and $30^{\prime}$ of the cluster center. This suggests that the $\mathrm{SB}$ activity must be related to the global properties of the cluster.

A question that remains is what triggered the star formation in the SB, and what stop it in PSBs? Several mechanisms have been suggested: galaxy-galaxy interactions, interaction with the cluster potential, and ISM-ICM interactions. The fact that the blue disk galaxies are $\mathrm{HI}$ deficient and have lost their gas in the outer parts, and the absence of optical disruptions, makes an ISM-ICM interaction more plausible. Thus we conclude that in Coma at least the starburst gets triggered by an interaction with the ICM and the starburst is likely to stop because most of the remaining gas gets swept out of the galaxies.

\section{References}

Bothun, G., and Dressler, A. 1986, ApJ, 301, 57

Bravo-Alfaro, H., Cayatte, V., van Gorkom, J.H., \& Balkowski, C. 2000, AJ in press

Caldwell, N., Rose, J. A., Sharpless, R. M., Ellis, R. S., and Bower, R. G. 1993, AJ, 106, 473

Caldwell, N., \& Rose, J. A. 1997, AJ, 113, 492

Donas, J., Milliard, B., \& Laget, M. 1995, A\&A, 303, 661

Dressler, A., \& Gunn, J. E. 1990, in Evolution of the Universe of Galaxies: Edwin Hubble Centennial Symposium. ed. R. Kron. San Francisco, Astronomical Society of the Pacific, p. 200

Vikhlinin, A., Forman, W., and Jones, C. 1997, ApJ, 474, L7 